\title{
The effects of binocular and motion-generated information on the perception of depth and height
}

\author{
MARGARET A. HAGEN \\ Boston University, Boston, Massachusetts 02215 \\ and \\ MARTHA TEGHTSOONIAN \\ Smith College, Northhampton, Massachusetts 01063
}

\begin{abstract}
The perception of distance and size in the presence of optical gradient information was investigated under four viewing conditions-binocular view with and without head motion, and monocular view with and without head motion. Subjects (60 adults) matched distance intervals (from 15 to $127 \mathrm{~cm}$ ) and heights of a target triangle (from 5 to $15 \mathrm{~cm}$ ) by adjusting the length of a metal tape. Both linear and power functions were fitted to each individual's distance judgments, and the competing perceptual models were compared. For both models, it was found that binocular information was sufficient to specify relative, but not absolute, distance, that monocular information was sufficient to specify an orderly relation between target distance and judgment but not absolute distance, that average error was less in the binocular conditions, and that perceived distance was not affected in either condition by the addition of head motion. The analysis of size judgments revealed that monocular and binocular judgments did not differ, that matches made with and without head motion did not differ, and that, in all conditions, matches exceeded target heights by an average $30 \%$ to $40 \%$. Judged size was also analyzed as a function of target distance. In all conditions but monocular view with head motion, the effect of distance was to increase size judgments. The distance judgments support the hypothesis (Purdy, 1958) that the binocular stimulus carries information that the monocular stimulus does not; they fail to support the hypothesis (Gibson, 1966) that observer motion adds information to the static stimulus. The size judgments support neither hypothesis but suggest an independence of perceived size from perceived distance.
\end{abstract}

The existence of trompe l'oeil demonstrates that a static monocular display may provide sufficient information to the observer to specify the spatial arrangement of objects in the pictured scene. The necessity for constraining the observer-for, when he views binocularly with free head movement, the deception is undone-demonstrates that additional information is provided by binocular parallax and motion parallax. The nature of that additional information is, however, at issue. It is clear that both binocular and motion parallax serve to differentiate flat from in-depth visual arrays. But, do they add additional information about size and distance relations when the observer views the in-depth array?

Gibson (1950) first suggested that the monocular static optical stimulus associated with an array of sur-

This research was supported by Grant R01 MH 27947-0 from NIMH to the first author, and the preparation of this report was supported in part by Grant 1RO HD10857 from NICHHD to the second author. We thank Rebecca Jones, Edward Reed, and Nancy Penniman, who ran subjects and aided in data analysis. Reprint requests should be sent to Martha Teghtsoonian, Clark Science Center, Smith College, Northampton, Massachusetts 01063. faces provided information about the spatial arrangement of those surfaces through distance-associated gradients (of texture, size, and compression of line). Purdy (1958) made a formal analysis of the geometry of the monocular optical stimulus, which assumes a physical environment made up of textured surfaces, one of which is a textured plane surface such as the ground or floor. He showed that the available information specified what he called "comparative" or relative distance - the case shown in Figure 1A, in which zero perceived distance corresponds to zero physical distance, and perceived distance ratios correspond to physical distance ratios, but perceived distance intervals do not necessarily correspond to physical distance intervals. In Figure 1A, judged distance $=.7$ (physical distance); if two target distances, of 5 and 10 in., are provided, judged distances will be 7 and 3.5 in., respectively. Whereas the real distance interval is 5 in., it is judged to be only 3.5 in., but the actual ratio of $10 / 5=2$ corresponds to the ratio of judged distances, $7 / 3.5=2$. This ambiguity of the monocular optical stimulus might be resolved, as Gibson (1966) suggested, by informa- 


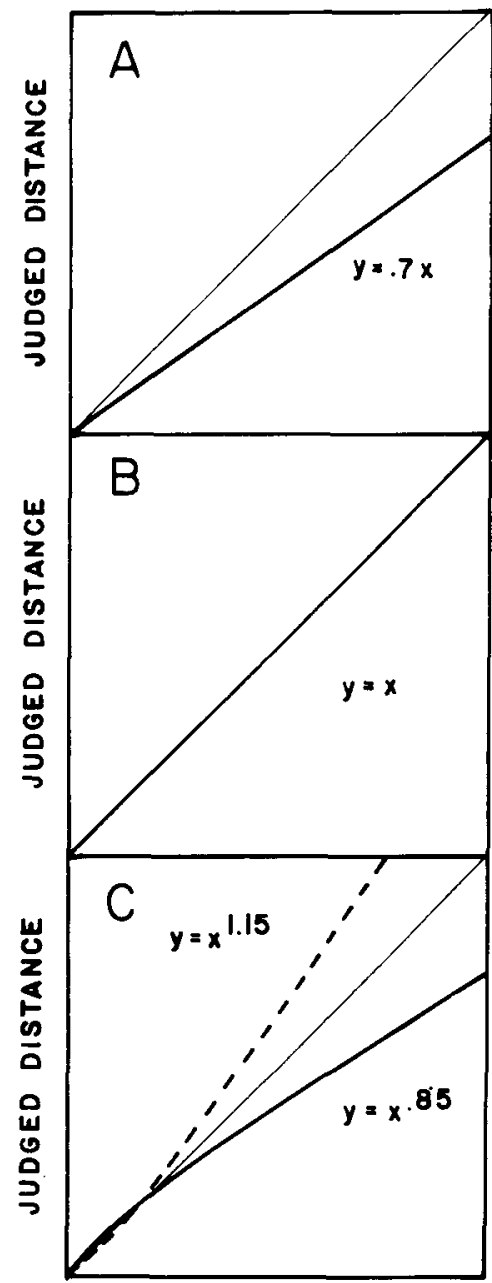

TARGET DISTANCE

Figure 1. Three possible relations between judged and target distances: (A) proportional, multiplicative constant not equal to 1; (B) proportional, multiplicative constant 1; (C) not proportional, since $y$ is a power function of $x$.

tion provided through movement of the observer relative to the array of surfaces; the gradient of velocities in the dynamic monocular stimulus might contain the information necessary to specify distance intervals and thus bring distance judgments into direct correspondence with actual distance, as shown in Figure 1B. Purdy (1958), however, showed that the same "comparative" information is specified by the monocular dynamic as by the monocular static stimulus. He argued, rather, that only the binocular static stimulus carries information adequate to specify the distance intervals, that is, to determine "absolute" distances. According to Purdy's analysis, when the optical slant of the ground surface and the base length are known, the absolute distance to a point on the surface is determined: In binocular viewing, the monocular display specifies the slant of the ground surface, and the interpupillary distance, a constant in binocular viewing, is incorporated into perceptual processes and specifies the base length."

Purdy's argument leads to the prediction that monocular viewing, whether with or without head motion, will produce judgments that are proportional to actual distance, but only when viewing is binocular will judgments move into one-to-one correspondence with actual distance. Gibson's view also predicts that the observer will make judgments of distance that are proportional to actual distance when viewing is monocular without head motion, but oneto-one correspondence will be achieved when head motion is added. The same arguments hold for judgments of size.

Both Gibson and Purdy assume that, under normal circumstances, an appropriate response measure will always be linearly related to physical distance and size. That this is not necessarily a safe assumption is indicated by the frequency with which the result shown in Figure 1C is obtained in psychophysical experiments, in which the response is shown to be a power function of the stimulus with an exponent different from 1; equal stimulus ratios produce equal response ratios, but the response ratio need not equal the stimulus ratio (for apparent distance functions that are not linear with physical distance, even though optical gradients were available to the observer, see Cook, 1978; Teghtsoonian \& Teghtsoonian, 1969). Gibson (1966) has argued that such results are confined to the measurement of the observer's response to stimulus energy, and that they are irrelevant to the pick-up of stimulus information. Nevertheless, the possibility of such a result should be borne in mind.

There are few studies that bear on the question of whether absolute size and distance information can be picked up from either the monocular dynamic optical stimulus or the binocular static optical stimulus. Distance judgments under monocular and binocular viewing were compared by Luria, Kinney, and Weissman (1967); however, they used a reduced-cue situation in which no optical gradients were available to the observer. Künnapas (1968) compared monocular and binocular distance judgments, but he, too, used a reduction situation. We can locate no studies that compared binocular with monocular distance judgments in a single (nonphotographic) setting that provided optical gradients.

The effectiveness of motion parallax as a determinant of relative perceived depth in the absence of any other distance information was first demonstrated by Gibson, Gibson, Smith, and Flock (1959). Gogel and Tietz (1979) used a one-object display and found that, in an otherwise dark field, the perceived distance of the object was modified in accordance with motion parallax cues. Using a twoobject display, Hell (1978) showed that, with motion parallax as the only effective cue to depth, relative 
sizes of the objects depended on depth, and the effectiveness of depth depended on velocity and amplitude of head motion, although, even at best, perceived depth produced a Brunswik ratio of .5 . Ferris (1972) showed that, with minimal cues to distance other than motion parallax, subjects could make distance judgments based on information generated by head movements, but these judgments were a decelerating function of actual distance; his stimulus situation provided only a target and background elements. None of these three studies provided a gradient of velocities, which Gibson's and Purdy's analyses specify, and thus are not appropriate tests of assertions that motion-generated information specifies either relative or absolute distance.

Two studies that do provide such gradients are those of Eriksson (1974) and Rogers and Graham (1979). Rogers and Graham used computer-generated random-dot patterns that simulated the relative movement information produced by three-dimensional corrugated surfaces when either the observer's head or the display oscilloscope moved from side to side. In each case, a compelling impression of threedimensionality, with appropriate depth relations, resulted; the authors conclude that "the amount of relative depth perceived and matched ... must depend on the accurate perception of both the absolute distance from the observer to the display and the translation path of the oscilloscope." Eriksson, using luminous patterns in an otherwise dark field, presented simultaneously several objects at several distances from the observer. When viewing was static and monocular, observers' reports indicated either that all objects were perceived in the same plane or that relative size and height in the picture plane determined perceived distance. Motion parallax induced by locomotion produced a perceptual reorganization into a veridical three-dimensional pattern, and absolute distance judgments were only slight underestimates of physical distance. Eriksson concluded that motion parallax may produce a "calibrated egocentric scale with regard to object distances."

That motion of some component of the visual field, relative either to the observer or to some other component as well, is not a sufficient condition for veridical distance perception is shown by at least two studies: Gogel and Tietz (1979) showed that, with a single light point as stimulus, motion parallax cues to distance could be canceled by cues from accommodation; Farber and McConkie (1979) showed that differential optical motions of two dot patterns produced a strong depth effect but that relative depth did not depend upon the relative velocities of the two patterns. Nonetheless, the findings reviewed here show that under some conditions motion parallax serves as an effective cue to relative depth relations, and that, when there is a gradient of velocities, motion parallax may provide cues for absolute distance.

Studies that compare apparent size as a function of distance under binocular and monocular viewing conditions with gradient-specified information include those of Leibowitz and Dato (1966) and Leibowitz, Pollard, and Dickson (1967). At distances up to $120 \mathrm{ft}$ indoors and $200 \mathrm{ft}$ outdoors, the same size matches were made by adults, regardless of viewing condition. These studies demonstrate the equivalence of monocular and binocular information for size matching, but were not designed to discover whether apparent size is specified absolutely or relatively.

The data available are thus not adequate to determine whether monocular viewing and head movement specify distance and size relatively, and binocular viewing specifies them absolutely, as Purdy (1958) has proposed. The purpose of the present study was to obtain distance and size scales under conditions in which optical gradients were available, to compare the effects of monocular with binocular viewing, with and without head movement. The viewing situation was a 2.4-m alley with black, white, and gray checked floor and white walls. In a procedure based on that used by Teghtsoonian and Teghtsoonian (1970), several different target heights were each presented at several different distances, and subjects made distance and size judgments by adjusting a metal tape to indicate the height of a target and its distance from a fixed marker.

\section{METHOD}

\section{Subjects}

Sixty men and women from introductory psychology classes served as subjects. There were 15 in each of the four conditions produced by a factorial crossing of monocular vs. binocular viewing with head motion present vs. head motion absent. About half of each group were male and half female.

\section{Apparatus}

The stimulus array contained a flat table top $4 \times 8 \mathrm{ft}(1.22 \times$ $2.44 \mathrm{~m}$ ) covered with black, white, and gray checked gingham (each square was $22 \mathrm{~mm}$ ) stretched tightly over 3/4-in. plywood. The table was surrounded by off-white curtains, $4 \mathrm{ft}(1.22 \mathrm{~m})$ high, suspended from the ceiling; the curtains prevented the observer from seeing the rest of the room. The targets were isosceles triangles cut from Day-Glo red-orange poster board and mounted upright with L-braces. A 2 -cm-diam red circle, placed flat on the table $18 \mathrm{in} .(45.8 \mathrm{~cm})$ from the edge nearest the observer, served as a marker from which target distance intervals were judged.

For all observers, viewing was through a large slot, 10.0x 6.0 in. $(25.4 \times 15.2 \mathrm{~cm})$, centered around the station point used in static viewing. The slot was cut into a cardboard screen, $4 \times 4 \mathrm{ft}$ $(1.22 \times 1.22 \mathrm{~m})$, mounted at the near end of the table. The entire checked surface, from the near end of the table to the curtain $8 \mathrm{ft}$ away, was visible to the observer. A subject in a monocular condition indicated the nonpreferred eye, which was covered with an eye patch. In the monocular condition without head motion, the eye was positioned 6 in. $(15.2 \mathrm{~cm})$ above the table and at 
the table's edge. In the binocular condition without head motion, the bridge of the nose was stationed at this point. Head motion was constrained in both head-motion-absent conditions by a chinrest with temple guides (Biometrika Model 115-4). In both headmotion-present conditions, there was no chinrest, and the subject was instructed to move head and shoulders freely from side to side while making each judgment, but to keep head in line with the triangle.

There were five target heights, represented by triangles that were 2.0, 3.0, 4.0, 5.0, and 6.0 in. high $(5.08,7.62,10.2,12.7$, and $15.2 \mathrm{~cm})$. There were five target distance intervals, measured from the circular marker to the base of the triangle; they were 6.0 , $10.2,17.3,29.5$, and 50.0 in. $(15.2,25.9,43.9,74.9$, and $127.0 \mathrm{~cm})$.

Each subject was run individually. The following instructions were read:

"This is an experiment in visual perception. We're interested in how people make judgments of size and distance.

"In front of you is a table and on the table there are two objects, a circle and a triangle. We are going to ask you two things: one, to estimate the size of the triangle, that is the distance from the base to the apex (height) and two, the distance between the two objects, that is, the distance from the center of the circle to the base of the triangle.

"You will make your judgments by using this tape measure. The procedure will be this: We will ask you for, say, a size judgment. You may look once through the peephole, but only once, and then try to duplicate the size of the triangle with the tape. The same thing will happen for distance. Look once, sit back, and pull out the tape to match the distance between the circle and the triangle. That is, if you were to lay the tape on the table after you make your judgment, one end would be at the circle, the other at the base of the triangle.

"Please hold the tape measure in your right hand and pull out the tape with your left so that you cannot see the numbers. Pull it out straight in front of you. When you've made your judgment, turn the tape on its side so that I can record your estimate. Then return the tape by pushing this button."

Each of the five triangle sizes occurred once at each of the five locations, and the subject judged both its distance from the circular marker and its height, for a total of 25 height and 25 distance interval judgments in 25 trials. Size judgments were requested first on half the trials, and distance judgments were requested first on the other half. This order was randomized, as was the order of the 25 height-distance combinations, for each subject. The 25 stimulus presentations were made without a break; the subject paced the presentation by determining the length of each look and the time to make a judgment. About $30 \mathrm{~min}$ was required to run each subject.

Judgments were made with a retractable 10-ft tape. The subject held the tape in the right hand and pulled out the tape, toward the left, to the matching length, holding it so that the inch markings were visible only to the experimenter. When the subject indicated a match, the experimenter recorded the length and the subject reset the tape to zero.

\section{RESULTS}

Figure 2 shows the median length matches to each distance interval under the four viewing conditions. (Medians were used to reduce the influence of an occasional deviant judgment; mean values show the same pattern of results.) In every case, the judged distance is less than the target distance. Monocular matches are lower than binocular ones; matches with head motion are lower than those without. The statistical evaluation of these differences requires a deci-

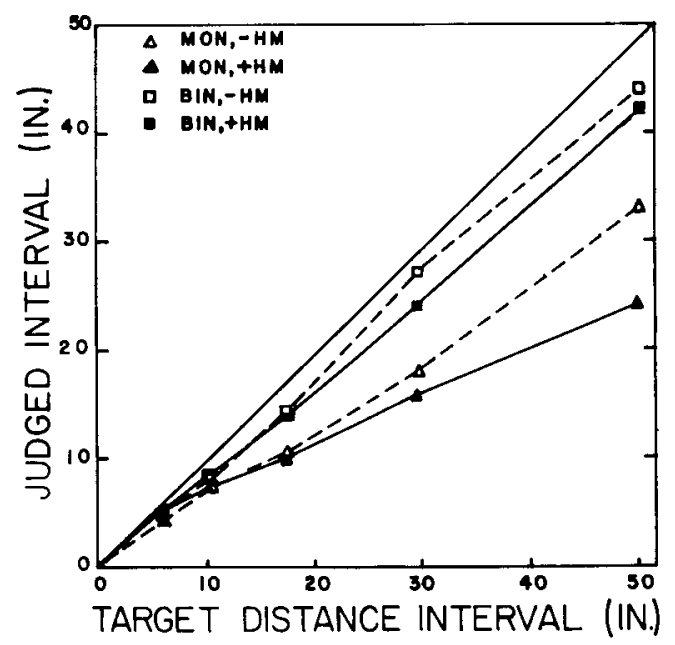

Figure 2. Median length match to target distance interval in four viewing conditions.

sion about whether the relation between judged and target distance should be modeled as a linear or as a power function. Two analyses are presented, with arguments for each.

\section{Judged Distance as a Linear Function of Target Distance}

Linear functions were fitted to each individual's distance judgments by the method of least squares, with the constraint that the function pass through the origin (equivalent to assuming that the subject understood that the red circular marker from which the distance interval was measured was zero). Table 1 shows the mean and standard deviation of individual slopes, as well as the mean percentage of variance accounted for by the fitted line, for each viewing condition. An analysis of variance of the individual slopes showed that the monocular vs. binocular difference is significant $[F(1,56)=13.75, p<.05]$, whereas neither the difference between absence and presence of head motion $[F(1,56)=1.85, p\rangle .05]$ nor the interaction $[F(1,56)<1]$ is significant. The distributions of slopes were combined for the monocular and for the binocular conditions. In each case, a $t$ test was used to evaluate whether the mean of the distribution differed significantly from a hypothetical mean of 1.00 . For monocular viewing, the mean slope of .65 differed from $1.00[t(29)=7.71$, $\mathrm{p}<.05]$; for binocular viewing, .87 differed from $1.00[t(29)=3.51, p<.05]$.

Thus, on the assumption that each subject's judgments of distance represented a linear function of target distance passing through the origin, (1) judgments made with binocular viewing have a steeper slope than those made with monocular viewing, (2) judgments made with head motion have the same 
Table 1

Mean and Standard Deviation of Slopes of Linear Functions Relating Individual Judgments to Target Distances, and Mean Percentage of Variance (V) Accounted for by Individual Functions

\begin{tabular}{|c|c|c|c|c|c|c|}
\hline \multirow{3}{*}{$\begin{array}{c}\text { Head } \\
\text { Motion }\end{array}$} & \multicolumn{3}{|c|}{ Monocular } & \multicolumn{3}{|c|}{ Binocular } \\
\hline & \multicolumn{2}{|c|}{ Slope } & \multirow[b]{2}{*}{ V } & \multicolumn{2}{|c|}{ Slope } & \multirow[b]{2}{*}{$\mathrm{V}$} \\
\hline & Mean & SD & & Mean & SD & \\
\hline Absent & .71 & .26 & 96 & .89 & .22 & 96 \\
\hline Present & .59 & .24 & 93 & .85 & .19 & 97 \\
\hline
\end{tabular}

slope as those made without head motion, and (3) with both binocular and monocular viewing, distance judgments are less than the target distances.

\section{Judged Distance as a Power Function of Target Distance}

Each subject's judgments were fitted by a power function, by regressing log length match on log target distance. The slope of the resulting linear function corresponds to the exponent of a power function, and its additive constant to the scale factor. That is, when $\log$ length $=\log k+b \log$ distance, then length $=k$ distance $b$. Depending on the values of $k$ and $b$, matches will be less than, equal to, or greater than target distances. Figure 3 shows this schematically. In Figure 3A, where $\mathbf{b}<1$, the relation between match and target is concave downward; in this case, the match equals the target at a distance of $\mathbf{4 0}$ in., smaller distances are overestimated, and greater distances are underestimated. For a fixed exponent, that value at which judged distance will equal target distance depends on the scale factor. In Figure 3C, where $b>1$, the function is concave upward-the point at which the match equals the target depending on scale factor-and here being 60 in., so that lesser distances are underestimated, and greater distances are overestimated. In Figure 3B, where $b=1$, a linear function is indicated; but again the scale factor can vary: When it is less than 1.0 , distances will be underestimated; when greater than 1.0 , distances will be overestimated. Thus, under- or overestimation depends both upon the particular combination of exponent and scale factor and upon the particular values chosen for targets.

Table 2 shows the mean and standard deviation of the distributions of individual exponents, as well as the percentage of variance (in log judgments) accounted for by the power function, for each viewing condition. An analysis of variance of individual exponents showed that the difference between monocular and binocular viewing is significant $[F(1,56)$ $=5.76, \mathrm{p}<.05]$, but the difference between moving the head and holding it still is not $[F(1,56)=1.05$, $p>.05]$, and the interaction is not $[F(1,56)<1]$.

The distributions of exponents were combined for the monocular and for the binocular conditions. For monocular viewing, the mean exponent of .86 is significantly different from a hypothetical value of 1.00 $[\mathrm{t}(29)=4.57, \mathrm{p}<.05]$, but for binocular viewing the mean exponent of .98 is not $[t(29)=.47, p>.05]$. An exponent of .86 for monocular viewing means that average judgments of distance deviate from linearity; the function relating judged to target distance is concave downward. The exponent of .98 for binocular viewing means that average binocular judgments of distance are linear with target distance (of individual subjects, 10 have exponents less than $1.0,8$ have exponents equal to 1.0 , and 12 have exponents greater than 1.0).

Because the scale factor is not independent of the exponent in these data (the correlation $r$ between individual exponent and scale factor is -.8 for monocular viewing, -.9 for binocular), differences in scale factor for head motion were evaluated sepa-

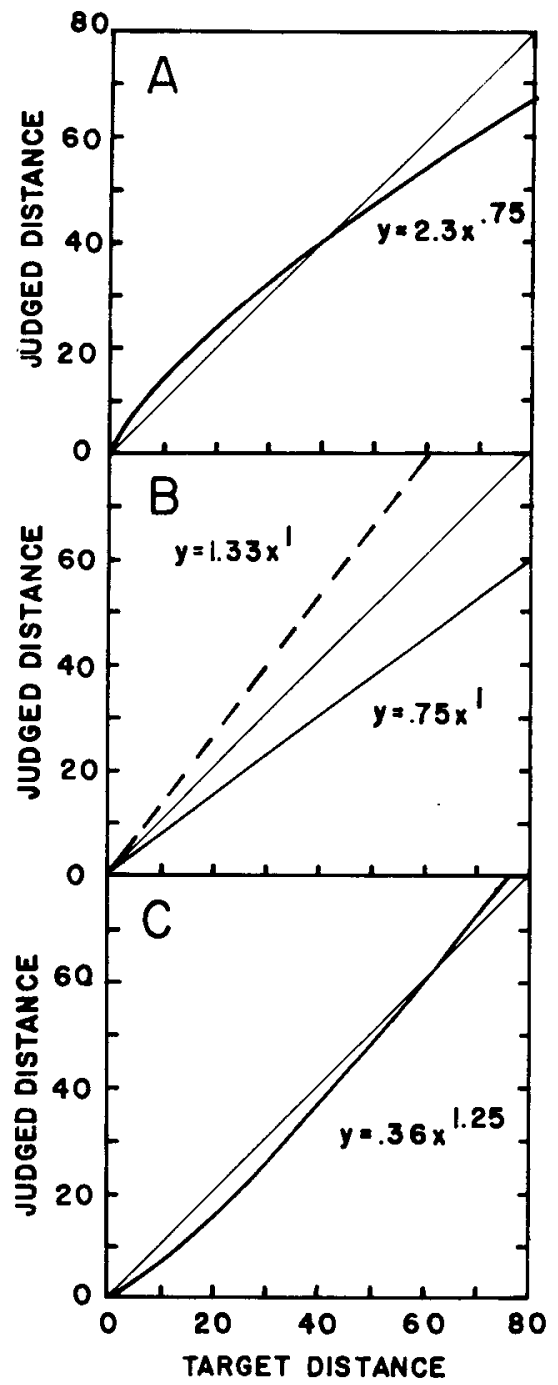

Figure 3. Hypothetical relations between judged and target distance. All are power functions: (A) exponent $\langle 1$; (B) exponent = 1; (C) exponent $>1$. 
Table 2

Mean and Standard Deviation of Exponents of Power Functions Relating Individual Judgments to Target Distances, and Mean Percentage of Variance (V) Accounted for by Individual Functions

\begin{tabular}{cccccccc}
\hline & \multicolumn{3}{c}{ Monocular } & & \multicolumn{3}{c}{ Binocular } \\
\cline { 2 - 3 } Head & \multicolumn{2}{c}{ Exponent } & & & \multicolumn{2}{c}{ Exponent } \\
Motion & Mean & SD & V & Mean & SD & V \\
\hline Absent & .91 & .14 & 98 & .99 & .21 & 98 \\
Present & .82 & .18 & 98 & .97 & .19 & 98 \\
\hline
\end{tabular}

rately for monocular and binocular viewing. For both, a Mann-Whitney $U$ test showed that the mean scale factor with head motion is not different from that without (for monocular, $U=89, p>.05$; for binocular, $U=100.5, p>.05$ ).

Are judgments of distance intervals equal to target distance intervals? For monocular viewing, the answer is clearly "no." Of the total 150 medians of distance judgments produced by 30 monocularly viewing subjects, only $13(9 \%)$-from 4 subjectswere greater than the target interval. For binocular viewing, the answer is also "no." Of the total 150 median distance judgments produced by 30 binocularly viewing subjects, only $31(21 \%)$ were greater than the target interval.

On the assumption that each subject's judgments of distance represent a power function of target distance, (1) judgments made with binocular viewing have a higher exponent than those made with monocular viewing, (2) the exponent for binocular viewing is 1.0, indicating no curvature in the function, whereas the function for monocular viewing is significantly concave downward, (3) judgments made with head motion have the same exponent as those made without head motion, and (4) with both binocular and monocular viewing, distance judgments are less than target distances.

\section{Linear vs. Power Functions}

Can we choose between modeling these data by linear functions and by power functions? The chief argument in favor of the linear model is conceptual simplicity: The results are easily describable in terms of changes in the slope constant and are most easily reconciled with a Gibsonian analysis of distance perception. The chief argument against the linear model is that it does not take account of the rather marked curvilinearity that is evident in the data of individual subjects: Two-thirds of subjects viewing under monocular conditions had functions that were concave downward, as did one-third of subjects under binocular conditions; one-third of subjects viewing under binocular conditions had functions that were concave upward. The fact that power functions were better fits than were the linear functions (see Tables 1 and 2 for comparable $r^{2}$ values) reflects the failure of the linear model in this respect.

As far as the purpose of this paper is concerned, analysis in terms of either model leads to the same conclusions. First, the information given by the monocular optical stimulus, although it is sufficient to specify an orderly relation between target distance and distance judgment, does not suffice to specify absolute distance. Second, the information given by the binocular optical stimulus is sufficient to specify relative, but not absolute, distance. Third, the average error in the binocular conditions is less than that in the monocular conditions, suggesting that additional information is picked up from the binocular stimulus. Fourth, perceived distance remains unchanged for both monocular and binocular viewing when motion-generated information is available, suggesting that no additional information for the present task is picked up from the dynamic optical stimulus.

\section{Judged Height as a Function of Target Height}

Target heights covered a range from 2 to 6 in., about half a $\log$ unit, and were not selected to discriminate between a linear and a power model: Over this small range, a linear function will provide an excellent fit and curvature will not be detectable. Therefore, the distributions of judgments were compared directly, instead of being fitted by linear or power functions.

Inspection of the distributions of length matches at each target height showed that they were positively skewed; consequently, the median was used as the measure of central tendency, and nonparametric statistics were employed.

Four subjects whose matches were patently deviant were discarded (three from the monocular without head motion condition, one from the binocular without head motion condition). These subjects assigned lengths that were over twice as great as the target heights, with some judgments three to six times as great; other subjects chose values between about equal and 1.8 times greater than the targets.

Figure 4 shows median length matches for the four conditions; the dotted lines indicate the interquartile range for the combined distributions. Well over $75 \%$ of the length matches fall above the target heights (indicated by the solid line $x=y$ ). The $x s$ show medians for the four discarded subjects.

Because some may feel uneasy about discarding subjects post hoc, the statistical analyses were performed on data from all subjects $(\mathrm{N}=60)$ as well as on the data without those from the four deviant subjects $(\mathrm{N}=56)$. A Kruskal-Wallis analysis of variance of the 56 ranked length matches for each target height showed no differences among conditions $[\mathrm{H}(3)<$ $7.82, p>.05]$, except for the 6-in. height $[H(3)=$ 


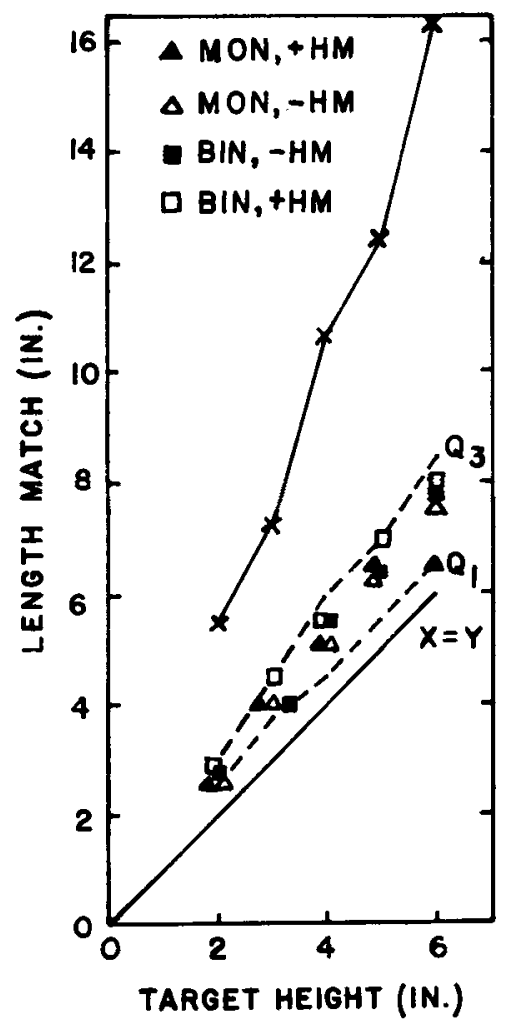

Figure 4. Median length match to target height in four viewing conditions. Dotted lines delimit the interquartile range. Xs show medians for four subjects whose data are not included in group function.

$9.08, \mathrm{p}<.05]$. For that height, the source of the significant difference was the median for monocular viewing with head motion, which was less than that for both binocular conditions $(z=2.530, p<.05$, by a one-tailed test for head motion present, and $z=$ $2.575, \mathrm{p}<.05$, by a two-tailed test for head motion absent). No other differences were significant. A Kruskal-Wallis analysis of variance of the 60 ranked length matches showed no differences among conditions $[\mathrm{H}(3)<7.82, \mathrm{p}>.05]$, except for the 6-in. height $[H(3)=9.42, p<.05]$. For that height, the source of the significant difference is the median for monocular viewing with head motion, which differs from the other three conditions $[U(15,15)<64$, $\mathrm{p}<.05$, by two-tailed tests]. In short, discarding data from four subjects does not alter the conclusions drawn from the statistical analysis, but the functions shown in Figure 4 are more representative of average performance than they would be if they included the discarded data.

We conclude from this analysis of the size judgments that (1) binocular matches do not differ from monocular matches, (2) matches made with head motion do not differ from those made without, and (3) in all conditions, matches exceed target heights by an average of $30 \%$ to $40 \%$.
The fact that length matches are greater than target heights may reflect some perceptual effect in this viewing situation or it may indicate the presence of a response bias. We can rule out the possibility that subjects tend, in general, to avoid low settings of the response tape, because length matches to target distances are smaller than the targets. In fact, the tallest height and the shortest distance are both 6 in., yet the tape is set $30 \%$ longer than the 6 -in. height but 20\% shorter than the 6-in. distance. Another possibility is that the vertical-horizontal illusion is contributing to the high matches to height, since the hand-held tape is horizontal, as are target distances, whereas target heights are vertical; at best, this might account for matches being $10 \%$ to $15 \%$ greater than target heights (Teghtsoonian, 1972).

The failure of size judgments to change when information from head motion is added to the static optical stimulus does not accord with Gibson's prediction. However, the failure to change when binocular information is added to the monocular optical stimulus does not accord with Purdy's prediction. It seems as if the size judgments are independent of perceived distance and are determined by other characteristics of the viewing situation, perhaps the context provided by the linear perspective or the artificial horizon (Sedgwick, 1973) of the background, which is unaltered by either of the experimental variables in this viewing situation. Leibowitz and Dato (1966) and Leibowitz et al. (1967) also found no differences in size matches for monocular vs. binocular viewing.

\section{Judged Height as a Function of Target Distance}

Each target height was presented at five distances from the subject. Figure 5 shows the effect of presentation distance on judged height, with responses pooled across targets by taking the mean of the

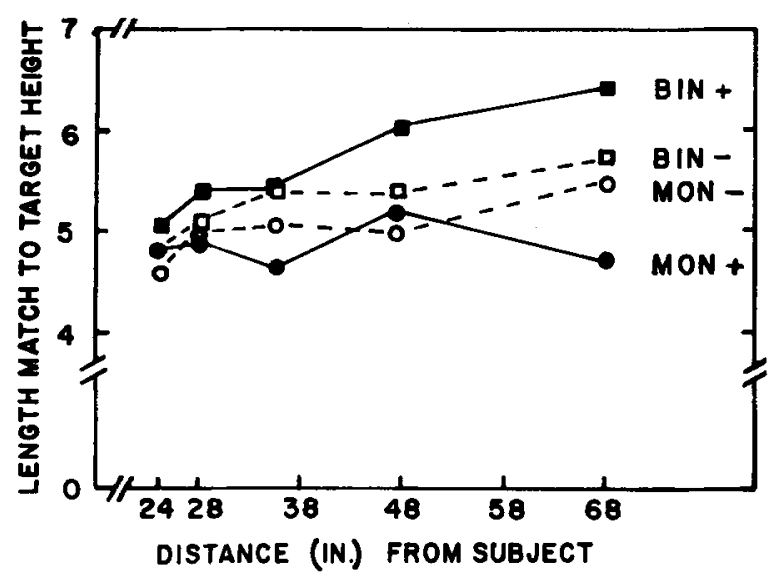

Figure 5. Length match to target height (pooled over five heights) at five distances from subject. 
Table 3

Significance of Difference Between Size Match at 6 and at 50 in. (by Wilcoxon Matched-Pairs, Signed-Ranks Test)

\begin{tabular}{|c|c|c|c|c|c|c|}
\hline \multirow[b]{2}{*}{ Condition } & \multirow{2}{*}{$\begin{array}{c}\text { Head } \\
\text { Movement }\end{array}$} & \multicolumn{5}{|c|}{ Target Height (in Inches) } \\
\hline & & 2 & 3 & 4 & 5 & 6 \\
\hline Monocular & $\begin{array}{l}\text { Absent } \\
\text { Present }\end{array}$ & $*$ & * & $*$ & & * \\
\hline Binocular & $\begin{array}{l}\text { Absent } \\
\text { Present }\end{array}$ & * & * & $\begin{array}{l}* \\
*\end{array}$ & * & * \\
\hline
\end{tabular}

${ }^{*} p<.05$, two-tailed test.

median length matches for the five target heights. For three viewing conditions, the effect of distance is to increase size judgments for a target of fixed size; for monocular viewing with head motion, distance has no effect on size judgments. These impressions are confirmed by statistical analysis: Table 3 shows the outcome of Wilcoxon tests of the difference between median size matches at 6 in. and at 50 in., which are predominantly significant at all heights for three viewing conditions, but not significant for the monocular with head motion condition.

Neither the finding that growth of matched size with distance is the rule in this viewing situation nor the finding that monocular viewing with head motion provides an exception to the rule was an expected finding. Both Purdy and Gibson assume that when adequate information is available in the optical stimulus, constancy should be the outcome; these results do not support that assumption.

The distance judgments can be used to predict the size judgments under the form of the size-distance invariance hypothesis that claims retinal size and apparent distance combine multiplicatively to determine apparent size: $k \theta d^{\prime}=s^{\prime}$, where $d^{\prime}$ is perceived distance and $s^{\prime}$ is perceived size. When apparent distance is linear with physical distance, size judgments should not change with distance: But both binocular conditions, in which the average exponent is 1.0 , show a growth of matched size with distance. When apparent distance is a power function of physical distance with an exponent less than 1.0, apparent size should decrease with distance: But, although the monocular distance exponent is $\mathbf{. 8 5}$, in one monocular condition matched size is invariant with distance, and in the other matched size increases.

The size-at-a-distance results, like the size judgments, are not predictable from the distance scales, a fact that supports the conclusion that size and distance judgments are independent in this viewing situation.

\section{DISCUSSION}

Judgments of distance intervals between a fixed marker and a variable target were less than the actual distance intervals. This was true, not only of median judgments, but also for $90 \%$ of individual subjects. However, the addition of binocular information to the monocular optical stimulus apparently enabled the subject to achieve distance perceptions that were closer to the actual spatial layout. This outcome is compatible with Purdy's (1958) analysis of information available in the optical stimulus. On the other hand, the addition of motion-generated information to the static optical stimulus produced no change in perceived distance, an outcome incompatible with Gibson's (1966) suggestion that motion parallax provides information for absolute distance scaling.

The curvilinearity of the individual distance functions (mostly concave downward in monocular viewing, but both downward and upward in binocular viewing) means that power functions are slightly better fits than are linear functions. This finding confirms that of Cook (1978), who, using partition procedures, constructed distance scales for individual subjects with exponents that ranged from values less than to values greater than 1.0.

Neither the absolute values of size judgments nor the size-distance functions were related systematically to distance judgments, since the smaller perceived distance in monocular viewing did not produce corresponding changes in size. In fact, the size judgments were independent of distance judgments, rather than appearing to be the results of a multiplicative correction of retinal size by apparent distance. This outcome is like that reported by Vogel and Teghtsoonian (1972), who showed that changes in apparent distance produced by introducing "false" perspective cues did not produce corresponding changes in perceived size.

\section{REFERENCES}

Cook, M. The judgment of distance on a plane surface. Perception \& Psychophysics, 1978, 23, 85-90.

Eriksson, E. S. Movement parallax during locomotion. Perception \& Psychophysics, 1974, 16, 197-200.

Farber, J. M., \& McConkie, A. B. Optical motions as information for unsigned depth. Journal of Experimental Psychology: Human Perception and Performance, 1979, 5, 494-500.

Ferris, S. H. Motion parallax and absolute distance. Journal of Experimental Psychology, 1972, 95, 258-263.

Gibson, E. J., Gibson, J. J., Smith, O. W., \& Flock, H. Motion parallax as a determinant of perceived depth. Journal of Experimental Psychology, 1959, 58, 40-51.

Gibson, J. J. Perception of the visual world. Boston: Houghton Mifflin, 1950.

Girson, J. J. The senses considered as perceptual systems. Boston: Houghton Mifflin, 1966.

GogeL, W. C. The metric of visual space. In W. Epstein (Ed.), Stability and constancy in visual perception: Mechanisms and processes. New York: Wiley, 1977.

Gogel, W. C., \& TIETz, J. D. A comparison of oculomotor and motion parallax cues of egocentric distance. Vision Research, $1979,19,1161-1170$.

HeLL, W. Movement parallax: An asymptotic function of amplitude and velocity of head motion. Vision Research, 1978, 18, 629-635. 